J Neurol Neurosurg Psychiatry 2004;75(Suppl II):ii31-ii36. doi: 10.1136/jnnp.2004.040501

T he management of low grade glioma is one of the most controversial areas in clinical neurooncology. There are numerous reviews and editorials outlining the difficulties in management of these lesions. ${ }^{1-3}$ Indeed, the pivotal questions about their management remain unanswered. However, the concept of management of low grade gliomas is not unitary but much more a composite of different challenges depending on the clinical presentation, signs, neuroradiology, perspectives of neurologists, the opinion of the neurosurgeon, and perhaps most importantly, the aspirations of the patient. It is true therefore that in many patients there will be a dilemma about what is considered optimal management since there is no good evidence base to underpin any single management undertaken. Conversely, however, there are many groups of patients with various low grade gliomas in whom management decisions are made more easily and pragmatically. In this review, the current approaches to different low grade gliomas presenting with different symptom complexes in different regions of the brain will be reviewed and the rationale for decision making discussed.

\title{
THE SPECTRUM OF LOW GRADE GLIOMA
}

Under the recent World Health Organization classification of primary intracranial tumours, low grade gliomas would encompass grade I and grade II neuro-epithelial tumours. The more common grade I tumours are pilocytic astrocytoma, dysembryoblastic neuro-epithelial tumours (DNET), pleomorphic xantho-astrocytoma (PXA), neurocytoma, and ganglioglioma. The more common grade II tumours include astrocytoma, oligodendroglioma, and mixed oligoastrocytoma. This spectrum of discreet neuropathological entities is important since the grade I tumours generally can be cured by surgical excision and their symptoms very often alleviated ${ }^{4}$ Conversely, with the grade II tumours, these are generally incurable but have median survival times of $>5$ years. ${ }^{1-3}$ Tumours with oligodendodrial components generally do better than astrocytomas, with prognosis being partially related to gene deletions on chromosome $1 \mathrm{p}$ and $19 \mathrm{q} .{ }^{5}{ }^{6}$ Some grade II gliomas are "diffuse" while others have relatively well defined brain-tumour interfaces. Neuropathological diagnosis and tumour characteristics will therefore profoundly influence the impact of treatment strategies. Currently, even with the best magnetic resonance imaging (MRI) scanners, differentiation between grade I, II, and even III tumours is very difficult, therefore establishing tissue diagnosis can be important. ${ }^{17}$

\section{WHAT SYMPTOM COMPLEXES DO LOW GRADE GLIOMAS CAUSE?}

While the median age of symptom onset is 35 years with grade II lesions, grade I lesions usually present at a younger age. The most common $(80 \%)$ presentation of a "low grade glioma" is with seizure disorders. This is particularly common with temporal, insular, frontal, and peri-rolandic lesions. Because in general these tumours displace or infiltrate the brain without destroying the neuropil, focal neurological deficits are uncommon (fig l). However, if the tumour has a cystic component, compression of adjacent cortical or white mater structures can lead to focal deficits (figs 2 and 3). Strategically placed lesions, often small ones, such as subependymal giant cell astrocytoma (seen with tuberous sclerosis) or intraventricular neurocytoma can also cause problems such as obstructive hydrocephalus. Lesions around the tectal plate, the foramina of Munro, and fourth ventricle will commonly present in this way.

With the increasing use of CT and MRI scans for investigation of "vague" neurological symptoms, an increasing number of low grade gliomas are being found incidentally.

Correspondence to: Professor lan R Whittle, Department of Clinical Neuroscience, Western General Hospital, Crewe Road, Edinburgh EH4 2XU, UK; irw@skull.den.ed.ac.uk

\section{Neuro-radiology}

Low grade gliomas are heterogenous and appearance will depend to some degree on the neuropathological diagnosis. Some lesions have intratumoural calcification; cysts may also be present and there is usually minimal peri-lesional brain oedema (figs 1-5). With MRI scanning, particularly with gadolinium enhancement, it is possible to be moderately confident of tissue diagnosis. However, in up to $30 \%$ of cases of what appears to be low grade gliomas are in fact 


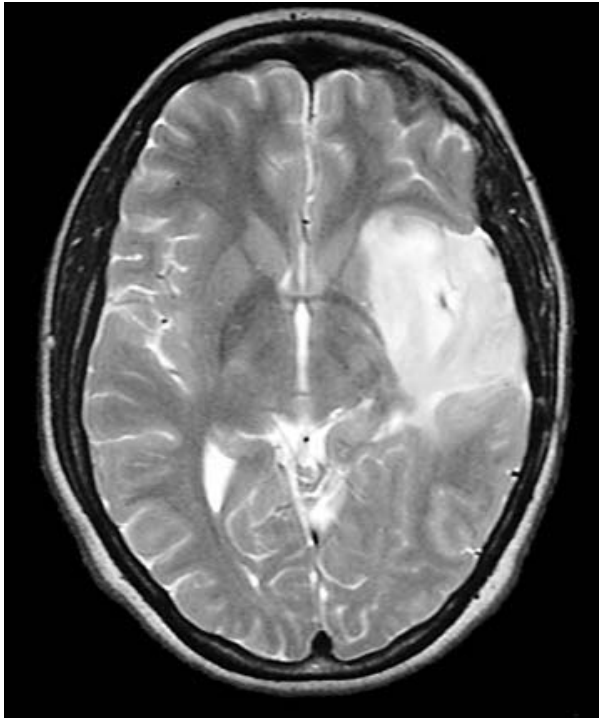

Figure 1 T2 weighted magnetic resonance image (MRI) scan of 28 year old woman with complex partial seizures but no focal neurological deficit. There is a hyper intense signal abnormality in the left temporal lobe and insular. The lesion did not contrast enhance and radiologically fits a classical appearance of a temporal-opercular-insular low grade glioma. This type of tumour more than any other characterises the dilemma in management of such patients. This woman's seizure disorder was controlled to some degree with anticonvulsants and she had a subtotal excision, which led to a diagnosis of oligodendroglioma grade II and complete cessation of her seizure disorder. Attempted complete excision of such lesions, even if performed using awake craniotomy techniques, can be followed by major neurological disabilities.

grade III or grade IV tumours. ${ }^{7}$ Spectroscopic analysis of the MRIs can help to clarify diagnosis but certainly not be definitive. Radiological diagnosis of a "low grade glioma" can therefore pose a dilemma.

There are two major advantages that have occurred with the advent of MRI scanning. Firstly, a policy of "watch and wait" can be introduced in some patients. The merits of this management strategy will be discussed later; however, it means that with good accuracy, the size and features of a lesion can be monitored serially with frequent scans. This is a distinct advantage over the less sensitive and radiation doses that are incurred with computed tomographic (CT) scanning. The other major advantage with MRI scanning is that the extent of the lesion can be evaluated using a range of sequences. The application of specific software programmes can enable precise volumetric analysis. Not infrequently, lesional enlargement occurs despite the scan apparently showing no obvious change in appearance between imaging periods. The third major advantage of MRI scanning is that it can be incorporated into image guided neurosurgical systems so that lesion localisation and, to some degree, excision can be facilitated during surgery.

\section{MANAGEMENT STRATEGIES IN LOW GRADE GLIOMAS}

Conventional strategies in neuro-oncology consist of surgery, radiotherapy, and chemotherapy. ${ }^{1-468-10}$ In addition, however, with low grade gliomas, because of the advent of MRI scanning, a "watch and wait" policy can be appropriate on occasions. ${ }^{2}{ }^{36}$ The raison d'être for the latter is that many lesions cause few symptoms for many years. This is
Symptoms and signs of low grade glioma

Epilepsy

- Headache

- Normal neurological examination

- Focal neurological deficits

Papilloedema

Neuro-endocrine disturbance

particularly the case in incidentally discovered lesions and those presenting with seizure disorders that are well controlled on anticonvulsants. If the lesion is surgically inaccessible, the patient does not wish to have surgery, or there are doubts about the evidence basis for surgery, then a "watch and wait" policy may be appropriate. If the patient's only symptom is seizure disorders, then generally these can be controlled to a variable degree with either monovalent or polyvalent anticonvulsant therapy. If the lesion enlarges, changes radiologically by developing foci of gadolinium enhancement, or symptoms or signs progress, then some form of intervention is usually required.

\section{WHAT CAN SURGERY OFFER?}

With modern surgical and anaesthetic techniques, many low grade gliomas can be completely or largely excised (fig 2-5). Grade I tumours causing seizure disorders that are refractory to anticonvulsants, or that the patient wishes to have excised, can generally be resected safely and with excellent control of the seizure disorder. The natural history of most grade I lesions is for any residuum of tumour not to grow and most of these patients can be considered "cured" (figs 2 and 3).

Most grade II lesions can also be resected to some degree. Complete neuro-radiological resection can be obtained in certain areas of the brain more readily than others. Polar and superficial lesions are more readily excised than deeper and more extensive lesions. There are particular hazards with surgery in certain locations. Low grade gliomas which extend into the temporal, insular or opercular regions pose a particular surgical challenge, since perforating branches of the middle cerebral artery may transverse the tumour to supply quite important white matter tracts in the centrum ovale $^{8}$ (fig 1). If these are damaged during surgical resection, then major deficit can result from the subsequent stroke. With the wider application of preoperative functional MR imaging (fMRI), a risk assessment exercise is possible. Additionally during surgery, use of awake craniotomy techniques mean that brain functions can be monitored and lesion excision optimised. However, even application of awake craniotomy technique is not infallible and permanent complications can occur. ${ }^{8-10}$

One of the important considerations when advising patients about surgery is that in the majority of cases surgery will not "cure" the tumour. Rather disappointingly, what tends to happen is that after many years of radiological quiescence or stability, tumours tend to recur several years later. ${ }^{1-3}$ They may recur locally or multifocally (fig 6). At this stage either further operation and radiotherapy or chemotherapy can be performed. However, given that in general there is a period of several years between diagnosis, with or without surgical intervention, and later relapse, a good dictum to guide initial management is "primum non nocere"-that is, "first do no harm". If ill advised, injudicious, or just unfortunate adverse events follow initial 


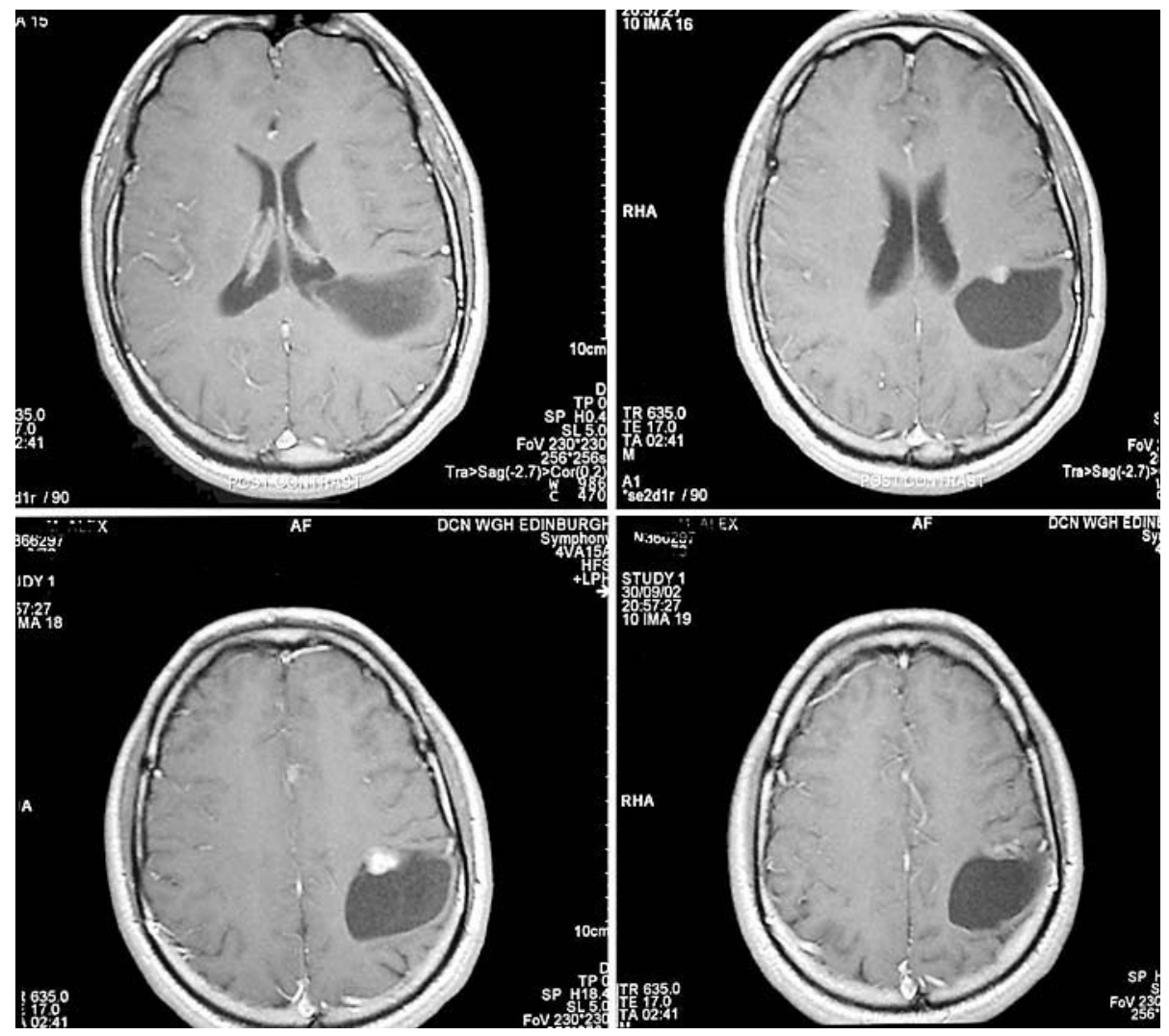

Figure $2 \mathrm{Tl}$ weighted gadolinium enhanced MRIs scans of a 30 year old man with a long standing history of seizure disorder but no focal

neurological deficit or headaches. The scans show a contrast enhancing neural module attached to the anterior wall of this left parietal lobe cyst. The lesion had been refractory to two anticonvulsants over many years.
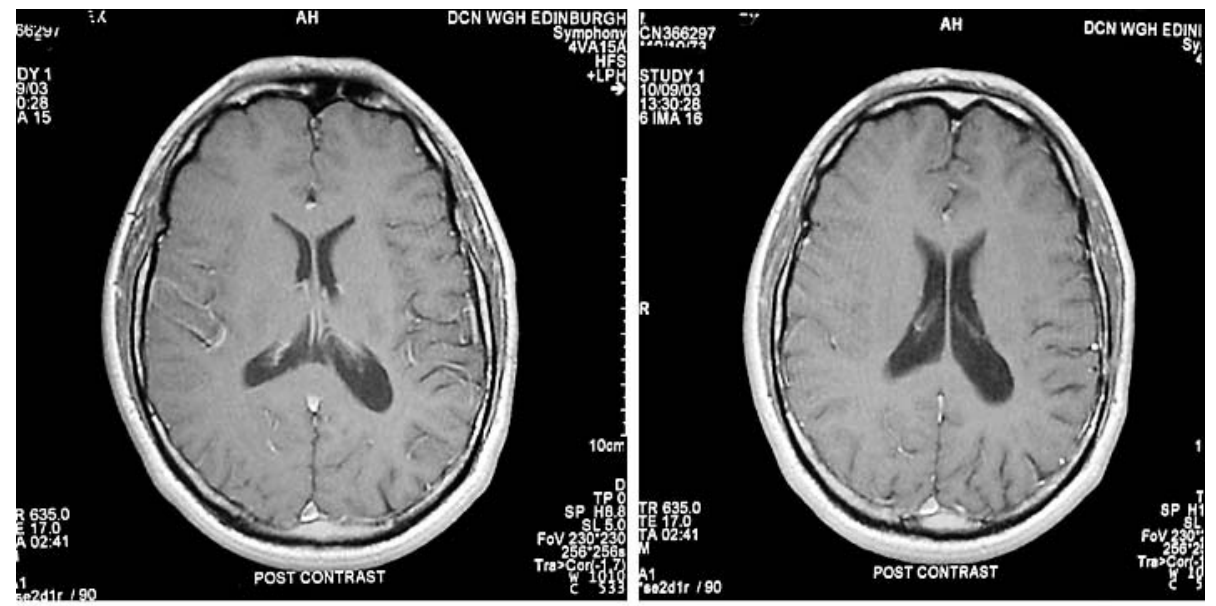

Figure 3 Following excision of the lesion shown in fig 2, pathology was found to be a pilocytic astrocytoma. The patient has been seizure-free since surgery and a postoperative MRI scan has shown complete re-expansion of the brain

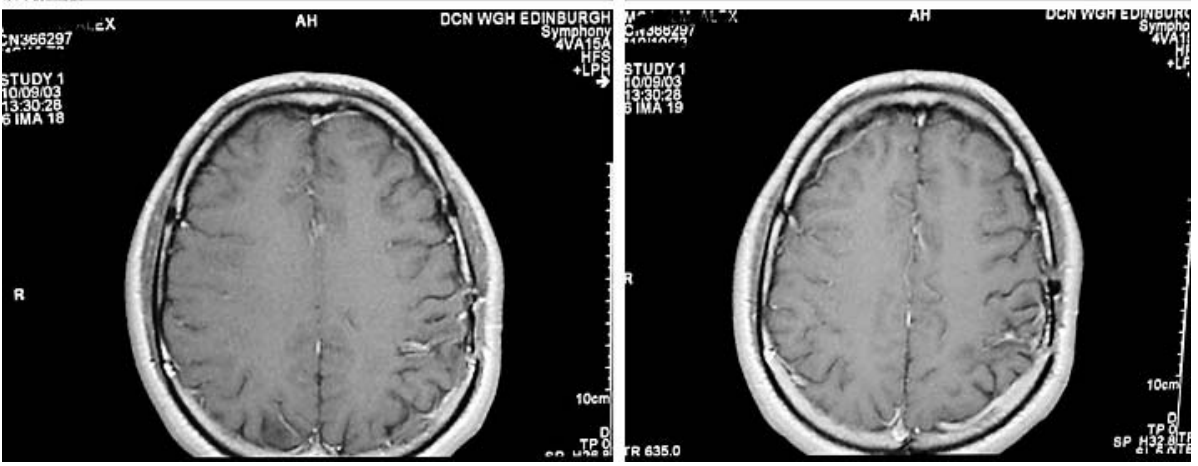




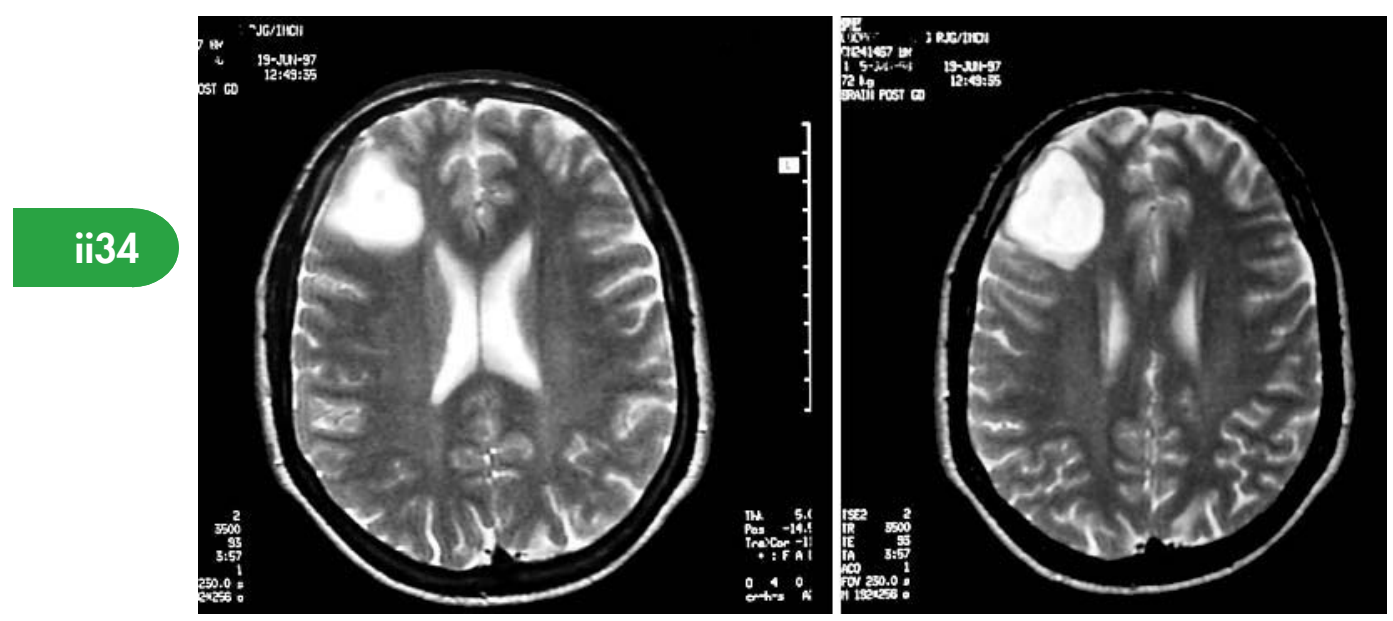

Figure 4 T2 weighted non-contrast MRI scans (see also fig 5) of a 41 year old man with a four year history of complex partial seizures. The seizure disorder had started four years earlier and serial MRI scans have shown progressive increase in the size of this lesion. It appears well defined. Because of the progressive increase in lesion size, the abnormality was excised in 1997. Pathology revealed an oligoastrocytoma.
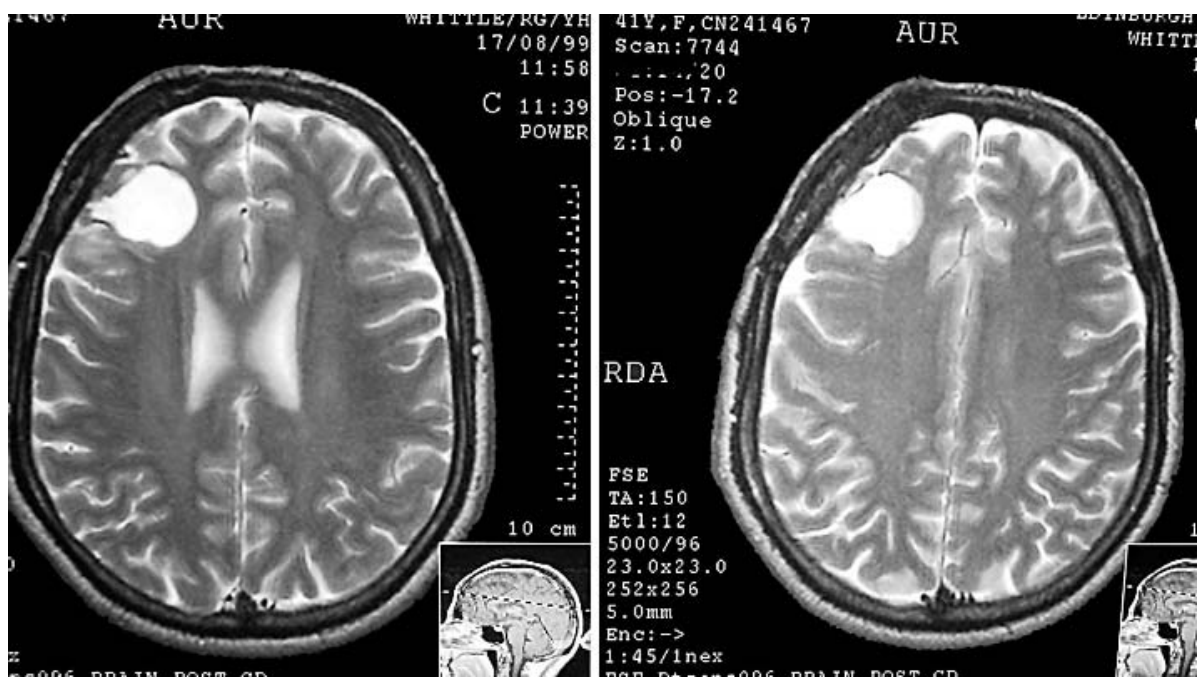

Figure 5 Postoperative T2 weighted non-contrast MRI scans of patient in fig 4 shows no residual tumour. The patient remained seizure free and completely well until 2004 when there was a local recurrence emanating from the posterior cyst wall and growing into the resection cavity.

surgery and these result in permanent neurological deficits, that patient has those deficits for the remainder of his or her life. Given that the vast majority of these patients tend to be young or working age and with families, infliction of such deficits can have major sociological and financial implications. In addition one is always reminded that they have been inflicted despite the surgery offering very little hope of cure. The likelihood of complications increases with the proximity of the lesion to eloquent cortex. If it is within $0.5 \mathrm{~cm}$ of functionally active and important brain, there is a high risk of complications. ${ }^{10}$ In many cases, however, deficits incurred following surgical resection using awake craniotomy techniques resolve over 1-3 months.

The major benefit of undertaking resective surgery or even biopsy is that a definitive diagnosis is obtained. ${ }^{1}$ In some cases biopsy may be all that is possible. Once a definitive diagnosis is made, then logical and appropriate radiotherapy and chemotherapy decisions and management can be undertaken. With biopsy, however, sampling is limited and there certainly can be focal anaplasia in what appears to be low grade gliomas. ${ }^{7}$

The benefit of surgical excision on survival in patients with low grade gliomas is not known. ${ }^{1-3}$ There are numerous case series that suggest the greater the amount of tumour excision the better the prognosis; that resection of neocortical tumours is more beneficial than resection of subcortical tumours; and that the smaller the volume of residual tumour the better the prognosis. Unfortunately, there has never been a randomised controlled trial of resection versus either biopsy or "wait and see". It is unlikely there will ever be such a study. However, there are hopes that with widespread application of molecular diagnostic techniques those grade II tumours with an "indolent" course can be differentiated from those with a more rapid progression. ${ }^{3}$ Such biological markers will hopefully have direct relevance to the use of radiotherapy and chemotherapy.

\section{WHAT IS THE ROLE OF RADIOTHERAPY?}

There have recently been several phase III studies evaluating the role of external beam radiotherapy in low grade gliomas. ${ }^{11-13}$ The studies were designed to determine what the optimum dose of radiotherapy is, and whether immediate radiotherapy is better than radiotherapy on symptomatic progression in patients with biopsy proven astrocytoma, oligodendroglioma, or oligoastrocytoma (grade II) tumours. Two studies ${ }^{11}{ }^{12}$ showed that higher dose treatment (59.4 Gy and $64.8 \mathrm{~Gy}$ ) did not improve upon the survival obtained with lower dose (45 Gy and $50.4 \mathrm{~Gy}$ ) therapy (five year survival $65 \% v 72 \%$ ). The overall survival at five years from diagnosis in early irradiated versus radiation at symptomatic progression was also similar $(63 \% v 66 \%)$, although time to progression was longer in the treatment group. ${ }^{13}$ 

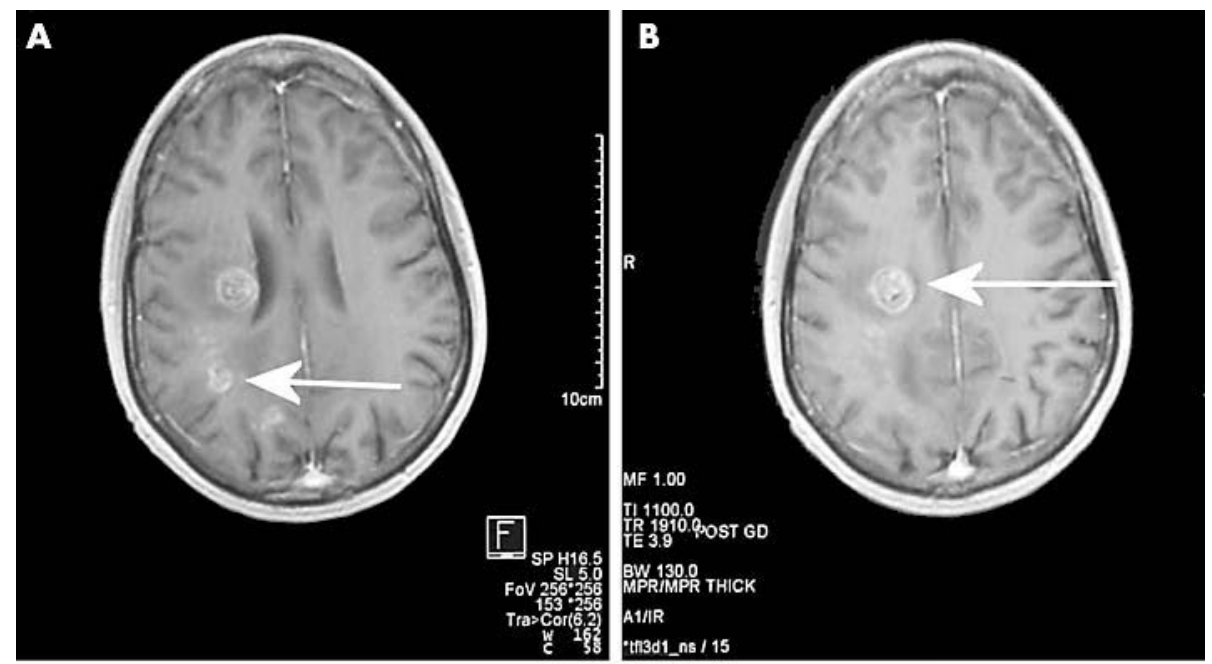

Figure 6 Recurrence of a glioma four years after excision using awake craniotomy technique. There is a recurrence in the white matter of the posterior and central semi ovale (panels $A$ and $B$, arrowed). Interestingly there has been minimal recurrence at the site of residual tumour (panel $\mathrm{C}$, arrowed) which was left following an awake craniotomy where the anterior part of the lesion was excised (panel D, arrowed). During the awake craniotomy functional activity was found to be present in the posterior part of the brain tissues and therefore this was not excised. This patient had several interval MRI scans showing stable residual disease over some years.
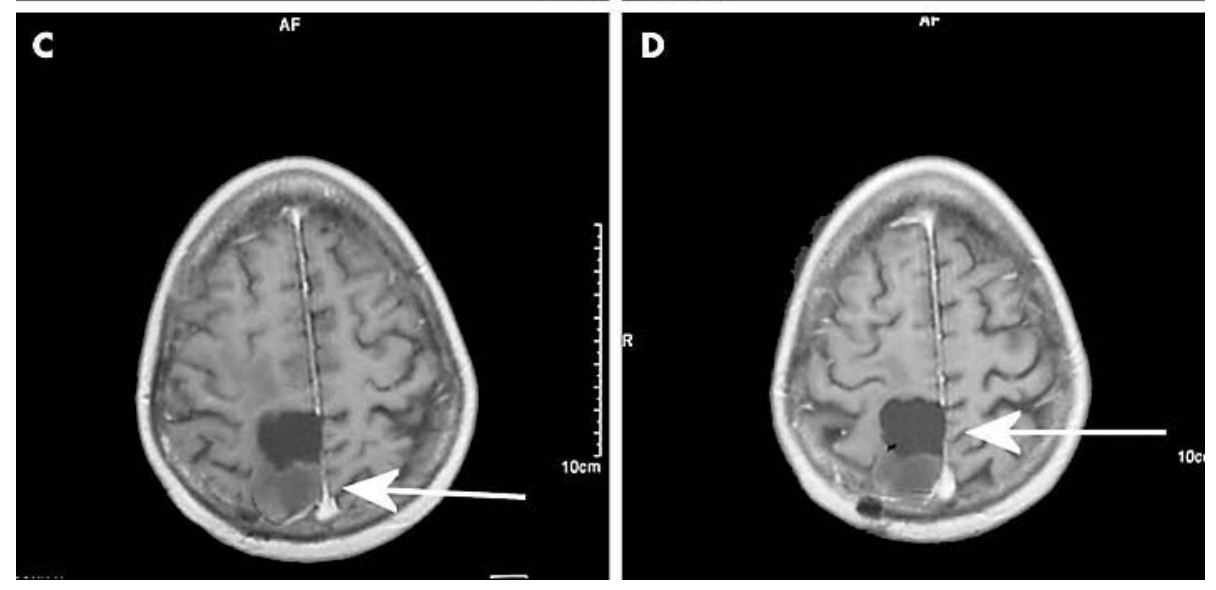

Not infrequently there can be a dramatic response in terms of volumetric shrinkage of the tumour following radiotherapy. These can also occur with interstitial radiotherapy and stereotactically delivered radiotherapy. Unfortunately, however, virtually all recurrences or progression of low grade gliomas occur in the irradiated areas. Although radiotherapy does not have the immediate risks associated with surgery, there are some longer term side effects as well as the immediate post-somnolence radiotherapy syndrome. ${ }^{14}{ }^{15}$ With the radiation doses used for low grade gliomas, radiotherapy can, if the field is large, induce a steady cognitive decline. This appears as progressive brain atrophy and white matter change on T2 MRI scan. Neuropsychological problems are aggravated by epilepsy and anticonvulsant therapy. ${ }^{15}$ As well as volumetric shrinking of the tumour, radiotherapy can also help control seizure disorders.

\footnotetext{
Management options in low grade glioma

- "Watch and wait"

Medical: anticonvulsants

- Surgical: image guided biopsy, image guided resection, \pm awake craniotomy with brain stimulation; cerebrospinal fluid diversion

- Radiotherapy, external beam, stereotactic radiosurgery, interstitial RT

- Chemotherapy; nitrosourea based, temozalamide
}

From the phase III radiotherapy studies prognostic features for low grade glioma were derived. Specific adverse features were: astrocytic glioma; midline shift; patient is aged over 40 years; and lesion is $>6 \mathrm{~cm}$ in diameter. ${ }^{16}$

\section{WHAT CAN CHEMOTHERAPY OFFER?}

Traditionally chemotherapy has been used for WHO grade III/ IV gliomas. However, because there is good evidence that some oligodendrogliomas with gene deletions of chromosomes lp and 19Q are chemosensitive, patients with WHO grade II oligodendrogliomas have also been treated with chemotherapy. ${ }^{5}$ In addition the advent of temozolimide, an alkylating agent that can be given orally, has also increased the attractiveness of chemotherapy. At this stage the only phase III trial evaluating chemotherapy has shown no benefit to survival in patients with incompletely resected grade II gliomas, ${ }^{17}$ and most other reports are anecdotal and often contradictory.

\section{WHY DO TREATMENTS FAIL?}

If grade I tumours such as PXA, DNET, neurocytoma, and pilocytic astrocytomas are excluded, conventional treatments will fail to cure the greater majority of WHO gliomas. The reason for failure of treatment is that most of these tumours have to some degree invaded the brain before diagnosis. Following observation, resection and/or radiotherapy, some of these quiescent cells later transform into more malignant phenotypes. The progression of low grade gliomas through a genetic catalogue of transformations to malignant glioma is 
Adverse prognostic features for patients with

low grade glioma

- Age $>40$ years

- Large tumour $(>6 \mathrm{~cm})$

- Midline shift

ii36

Neurological deficit

well documented. ${ }^{3}$ Unfortunately, the triggers for these transformations and the timing of them is poorly understood. In addition, at present there is no known treatment that can delay or stop this genetic de-differentiation. Until a greater understanding of these molecular mechanisms is gained, current treatments are taking a "blunderbuss" approach and causing variable "co-lateral" damage to the brain. Nonetheless targeted treatment in selected patients with adverse risk factors should do more benefit than harm.

\section{PATIENT PERSPECTIVES}

Because of the wide availability of a huge amount of information concerning gliomas on the world wide web, new patients are now particularly well informed, albeit not infrequently with poor quality information. I believe it is important to discuss uncertainties in management decision making with patients and involve them in decision making about their future. In my experience there are several stereotypic patient groups. Firstly the group that abrogates any contribution to decision making and adopts the approach that "doctor knows best". This calls on the experience and wisdom of the treating neuro-clinician since there is little evidence base for management decisions in this group of patients. Clearly, however, if a lesion is enlarging, causing focal neurological deficit or signs and symptoms of raised intracranial pressure or intractable epilepsy, a decision to operate is relatively straightforward. However, where epilepsy is well controlled and the lesion is static, there may be no compelling argument for surgery.

The second group of patients tend to be those in whom if surgery is possible and available, they want something done. This conviction will be held despite an explanation that there may be risks involved with surgical excision, and the natural history of the disease following even apparent complete surgical excision may not be altered by this approach. Another group of patients are those who are particularly well informed, understand the difficulties in management and the shortcomings of all treatments, and are particularly aware of problems that may need surgical or other interventions. There is also a more problematic fourth group who go around seeking several expert opinions in an attempt to answer a dilemma which cannot be solved.

\section{REFERENCES}

1 Keles GE, Lamborn KR, Berger MS. Low-grade hemispheric gliomas in adults: a critical review of extent of resection as a factor influencing outcome. J Neurosurg 2001:95:735-45.

2 Wilson CB, Bramwell V, Bonfill X, et al. Central nervous system tumours. In: Williams C, ed. Evidence-based oncology. London: BMJ, 2003:561-4.

3 Wessels PH, Weber WE, Raven G, et al. Supratentorial grade II astrocytoma: biological features and clinical course. Neurology. Lancet Neurol 2003;2:395-403.

4 Luyken C, Blumcke I, Fimmers R, et al. The spectrum of long-term epilepsyassociated tumors: long-term seizure and tumor outcome and neurosurgical aspects. Epilepsia 2003:44:822-30.

5 Ellis TL, Stieber WW, Austin RC. Oligodendroglioma. Curr Treat Options Oncol 2003;4:479-90.

6 Feigenberg SJ, Amdur RJ, Morris CG, et al. Oligodendroglioma: does deferring treatment compromise outcome? Am J Clin Pathol 2003;26:e60-6.

7 Scott JN, Brasher PM, Sevick RJ, et al. How often are nonenhancing supratentorial gliomas malignant? A population study. Neurology 2002;59:947-9.

8 Lang FF, Olansen NE, DeMonte F, et al. Surgical resection of intrinsic insular tumors: complication avoidance. J Neurosurg 2001;95:638-50.

9 Duffau H, Capelle L, Denvil D, et al. Functional recovery after surgical resection of low grade gliomas in eloquent brain: hypothesis of brain compensation. J Neurol Neurosurg Psychiatry 2003;74:901-7.

10 Peraud A, Meschede M, Eisner W, et al. Surgical resection of grade II astrocytomas in the superior frontal gyrus. Neurosurgery 2002;50:966-75.

11 Karim AB, Maat B, Hatlevoll R, et al. A randomized trial on dose-response in radiation therapy of low-grade cerebral glioma: European Organization for Research and Treatment of Cancer (EORTC) Study 22844. Int J Rad Oncol Biol Phys 1996;36:549-56.

12 Shaw E, Arusell R, Scheithauer B, et al. Prospective randomized trial of lowversus high-dose radiation therapy in adults with supratentorial low-grade glioma: initial report of a North Central Cancer Treatment Group/Radiation Therapy Oncology Group/Eastern Cooperative Oncology Group study. J Clin Oncol 2002;20:2267-76

13 Karim AB, Afra D, Cornu P, et al. Randomized trial on the efficacy of radiotherapy for cerebral low-grade glioma in the adult: European Organization for Research and Treatment of Cancer Study 22845 with the Medical Research Council study BRO4: an interim analysis. Int J Rad Oncol Biol Phys 2002;52:316-24.

14 Brown PD, Buckner JC, O'Fallon JR, et al. Effects of radiotherapy on cognitive function in patients with low-grade glioma measured by the folstein minimental state examination. J Clin Oncol 2003;21:2519-24.

15 Klein M, Heimans JJ, Aaronson NK, et al. Effect of radiotherapy and other treatment-related factors on mid-term to long-term cognitive sequelae in lowgrade gliomas: a comparative study. Lancet 2002;360:1361-8.

16 Pignatti F, van den BM, Curran D, et al. European Organization for Research and Treatment of Cancer Brain Tumor Cooperative Group, European Organization for Research and Treatment of Cancer Radiotherapy Cooperative Group. Prognostic factors for survival in adult patients with cerebral low-grade glioma. J Clin Oncol 2002;20:2076-84.

17 Eyre HJ, Crowley JJ, Townsend JJ, et al. A randomized trial of radiotherapy versus radiotherapy plus CCNU for incompletely resected low-grade gliomas: a Southwest Oncology Group study. J Neurosurg 1993;78:909-14. 